\title{
Optimización de Residuos Mineros de Carbón para el Mejoramiento de Suelos Viales con Algoritmos Evolutivos Multiobjetivo
}

Yolanda A. Jiménez ${ }^{(1) \star}$, Marlon J. Bastidas ${ }^{(2)}$ y José L. Consuegra(1)

(1) Facultad de Ingeniería y Ciencias Básicas, Dpto. de Ingeniería Civil, Fundación Universitaria del Área Andina, Centro de Investigación y Desarrollo, Avenida Circunvalar, Sede Valledupar - Colombia.

(e-mail: yjimenez25@areandina.edu.co; joconsuegra@areandina.edu.co)

(2) Facultad de Ingeniería, Grupo DESTACAR, Dpto. de Ingeniería Mecánica, Universidad de La Guajira,

Km 5 - Vía a Maicao, Riohacha - Colombia. (e-mail: marlonjoseb@uniguajira.edu.co)

${ }^{*}$ Autor a quien debe ser dirigida la correspondencia

Recibido Oct. 24, 2018; Aceptado Dic. 27, 2018; Versión final Feb. 5, 2019, Publicado Ago. 2019

\section{Resumen}

En este estudio se desarrolló un Algoritmo Evolutivo Multi Objetivo (AEMO) para determinar las mezclas optimas de material estéril extraídos de la minería del carbón a cielo abierto con cal y cemento, para su uso en construcción. El objetivo es compensar el efecto ambiental de la extracción de estos materiales de la minería del carbón aplicándolos en la estabilización de suelos para infraestructura vial. Se establecieron dos funciones objetivo: maximizar la capacidad de soporte del suelo y minimizar los costos, con restricciones asociadas a la granulometría, plasticidad y costos de los aditivos. Se muestra que estos materiales pueden ser utilizados para material de afirmado, subbase y/o terraplén con un tratamiento previo de la granulometría y un ajuste de los porcentajes de cal (entre el 2 y el $4 \%$ ) y cemento (menos del 1\%) mediante la aplicación del AEMO, quedando la Relación de Soporte California (CBR) e índice de plasticidad dentro del rango adecuado.

\section{Obtaining of Coal Mining Waste Mixtures for the Soil Stabilization Through Multiobjective Evolutive Algorithm}

\begin{abstract}
In this study a Multi-Purpose Evolutionary Algorithm (AEMO) was developed to determine the optimum mixtures of these materials with lime and cement to use these mixtures as construction material. The objective is to compensate for the environmental impact of the extraction of these materials of carbon open mines, applying the mixtures to stabilize roads. Two objective functions were established: to maximize soil support capacity and minimize costs, with restrictions associated with granulometry, plasticity and additive costs. It is shown that these materials can be used for bond material, subbase and/or fill with a pre-treatment of the granulometry and an adjustment of the percentages of the lime. (between 2 and $4 \%$ ) and cement (less than 1\%) by applying the AEMO, leaving the California Bearing Ratio (CRB) and plasticity index within the appropriate range.
\end{abstract}

Keywords: excavation material; industrial reuse; mining; evolutionary algorithms; optimization 


\section{INTRODUCCIÓN}

Los residuos mineros de carbón se han venido utilizando en diferentes aplicaciones; países como Alemania, España, Polonia, República Checa y Francia destinan millones de toneladas al año de estos residuos como material de relleno para carreteras y terraplenes (Fernández, 1995). En Colombia se realizó un estudio sobre el desempeño mecánico de morteros con adición de cenizas volantes procedente de la combustión del carbón, obteniendo un producto con especificaciones ajustadas a las Normas Técnicas Colombianas (Morales, 2015), en la misma temática Mas et al (2016) en Madrid (España) realizan un análisis del impacto ambiental producido por la utilización de morteros cuyo ligante son desechos de polvo de vidrio, con el fin de minimizar el impacto negativo en el medio ambiente a la hora de su puesta en obra mediante la utilización de materiales de construcción que reutilicen productos de desecho. Las metodologías de optimización se han utilizado en diversos proyectos, tales como viales, energía, diseño de mezclas, costos, etc., especialmente empleando algoritmos bioinspirados. (Bastidas et al, 2010). En este caso, la metodología más utilizada es el análisis multiobjetivo, que es una herramienta de programación aleatoria que halla la mejor solución de un sistema, teniendo en cuenta múltiples funciones objetivo. George Polya fue uno de los pioneros de los métodos de optimización multiobjetivo, con métodos para resolver problemas a través de la heurística. Los Algoritmos Evolutivos surgen para mejorar la eficiencia heurística, es decir, hallar la solución a un problema empleando la menor cantidad de recursos (Ramírez, 2014).

Trivedi et al (2013) formularon un modelo basado en un algoritmo genético que se puede usar para predecir la variación en los valores de CBR en subbases con la adición de un porcentaje específico del mismo residuo, permitiendo a todo tipo de agencias involucradas en la construcción de carreteras a predeterminar la estabilización de un suelo mediante el uso de estos residuos. Taskiran (2010) presenta una de las primeras investigaciones que tiene como objetivo investigar la aplicabilidad de los métodos de inteligencia artificial para la predicción del CBR en suelos de grano fino teniendo en cuenta diferentes propiedades como índice de plasticidad, contenido de humedad óptimo y límite líquido. Rojas et al (2016) presentan un modelo de asignación óptima de presupuesto para mejoramiento de calidad del servicio en sistemas de distribución, el cual consiste en un problema multiobjetivo que busca al mismo tiempo minimizar el costo de mantenimiento en estos sistemas y maximizar la reducción de la tasa de fallas.

En el campo de la ingeniería civil, los primeros trabajos encontrados con AEMO, fueron desarrollados por Coello en 1997, optimizando el diseño de vigas de hormigón armado, sometidas a restricciones específicas, teniendo en cuenta factores como el costo de materiales y el costo de procesos de producción. (Coello et al, 1997). Chau, et al (2003) crean una aplicación para la optimización de depósitos rectangulares de hormigón; Lepš, et al (2003) aplican un algoritmo para la optimización de una viga continua simétrica de hormigón utilizando una población de soluciones; Lee (2003), propone optimizar pórticos de planos de hormigón armado empleando un algoritmo evolutivo con una estrategia elitista; Fairbairn et al (2004) diseñan un procedimiento para la optimización de construcción de estructuras de hormigón utilizando como variables de entrada, el tipo y costo de los materiales, resistencia térmica y distribución interna de cada componente, lo cual permitió estimar características de funcionamiento de la estructura. Lim et al (2004) ensayan el balance óptimo de proporciones en la fabricación de hormigón de alta resistencia; Amirjanov et al (2005) utilizan un algoritmo evolutivo para la optimización de las proporciones de materiales compuestos de cemento y hormigón teniendo en cuenta el rendimiento y propiedades físicas del material; Saffon (2005) desarrolla una herramienta computacional basada en un Algoritmo Genético como soporte en toma de decisiones en la etapa de planeación de proyectos con el fin de ofrecer una programación de obra ajustada y comprobar su aplicabilidad.

Por otra parte, Chisari et al (2015) aplican un enfoque de identificación basado en algoritmo genético al estudio de caso de un puente de hormigón postensado aislado en una base en condiciones de carga estática y dinámica, el sistema estructural examinado proporciona una identificación estructural eficiente y bien calibrada de sus propiedades en comparación con otras herramientas de identificación y técnicas de correlación clásicas, permitiendo estimar un mayor número de parámetros en las propiedades del concreto. Gandomi (2018) utilizan tres tipos de algoritmos evolutivos para el análisis y diseño óptimo de un muro de contención de hormigón en voladizo bajo carga sísmica considerando medidas, costo y peso mínimo, mostrando los mejores resultados en todos los estudios de caso. Tiachacht et al (2018) presentan una nueva metodología para la identificación y cuantificación de daños en estructuras mediante la aplicación de un algoritmo genético, los resultados del análisis mostraron que la técnica propuesta es precisa y eficiente, en comparación con otras técnicas de la literatura, para estimar la severidad del daño estructural. Bastidas et al. (1993) determinaron las características mineralógicas y cerámicas de las arcillas mediante análisis mineralógico-petrográficos, químicos, granulométricos y límites de Atterberg para definir su posibilidad de utilización para la fabricación de pavimentos. 
Finalmente, Đumić et al (2018) implementaron un algoritmo genético para la planificación de proyectos con recursos limitados a lo largo de diferentes períodos de tiempo, los principales resultados mostraron que estos son una opción viable cuando hay una necesidad de programación personalizada en un entorno dinámico. Torres (2015) desarrolló una herramienta de optimización heurística multiobjetivo para la evaluación de alternativas de conservación que permita la asignación más sostenible y eficiente de recursos para la conservación de redes de transporte terrestre urbano en Chile. Korkana et al (2016) presentan un estudio donde se utilizan los algoritmos genéticos a través de EPANET y MATLAB para ofrecer una solución óptima de la red de distribución de agua con el desarrollo de una función objetivo precisa. Kanumuri et al (2017) implementaron un algoritmo genético para predecir las propiedades mecánicas de un tipo de acero inoxidable a temperaturas elevadas. Los resultados mostraron que el modelo matemático permite acelerar la velocidad de convergencia y resolver el problema del mínimo local para realizar la búsqueda global.

Esta investigación aporta soluciones a la disposición de los residuos de la minería para aplicarlos en el campo de la infraestructura vial. Para esto se plantea como objetivo principal desarrollar una herramienta para la evaluación de alternativas de optimización AEMO (Análisis Evolutivo Multi Objetivo) que permita obtener una dosificación de aditivos para mejorar las propiedades físicas y mecánicas según lo planteado por Gómez et al (2004) de mezclas de residuos mineros, como una nueva alternativa para ser utilizado como material de afirmado, subbase y terraplén, entre otras posibles utilizaciones. Finalmente, es importante resaltar que este trabajo está apoyado por la Red de Investigación Tecnológica en Nuevos Materiales y Procesos Constructivos para Infraestructura Vial - INNOVIAL, la cual investiga el aprovechamiento de residuos generados en el territorio nacional y su posible utilización en el campo de la infraestructura vial.

\section{METODOLOGÍA}

Inicialmente se tomaron cinco muestras de material residual de la mina de carbón Cerrolargo ubicada en el Municipio de La Jagua de Ibiríco (Cesar) con el fin realizar una caracterización del material para su posible uso como afirmado, subbase y terraplén.

La Tabla 1 muestra los ensayos realizados a las muestras de residuos y los equipos utilizados según las normas de ensayos del INVÍAS. El ajuste granulométrico fue programado con el fin de obtener la mezcla ideal de agregados que satisfaga los requisitos para todos los tamices establecidos para ser utilizados como material de afirmado, subbase y terraplén.

Tabla 1: Ensayos realizados a las muestras de residuo.

\begin{tabular}{|l|l|l|}
\hline Ensayo & Norma & Equipo \\
\hline Densidad real y aparente & INV-E-222/223-13 & Picnómetro de helio modelo ACUPYC \\
\hline Área superficial & NA & Gemini VII 3.03. Se empleó el método BET. \\
\hline Conductividad eléctrica & NA & Conductímetro multiparámetro HQ 40 D \\
\hline Humedad & NA & norma ASTM D2216 \\
\hline Difracción de Rayos X & NA & $\begin{array}{l}\text { Difractómetro de Rayos X marca PANALYTICAL } \\
\text { EMPYREAN modelo 2012 en corridas de 7 a 80 en 20. }\end{array}$ \\
\hline Fluorescencia de Rayos X & NA & $\begin{array}{l}\text { Espectrómetro marca THERMO modelo OPTIM'X (FRX) con } \\
\text { pastillas KBr }\end{array}$ \\
\hline Granulometría & INV-E-122-13 & Serie de tamices marca ENDECOTTS \\
\hline Límites de Atterberg & INV-E-125/126-13 & Cazuela de Casagrande y demás equipos de ensayo. \\
\hline Resistencia al desgaste & INV-E-218-13 & Máquina de los Ángeles. \\
\hline Sanidad de los agregados & INV-E-220 & Horno, balanza, soluciones de sulfato de sodio \\
\hline Proctor Modificado & INV-E-142-13 & Moldes, martillo, balanza. \\
\hline CBR & INV-E-148-13 & HM 3000, marca HUMBOLDT \\
\hline Ajuste granulométrico & NA & Programado \\
\hline
\end{tabular}

\section{Planteamiento del problema multiobjetivo}

Una vez obtenidos los resultados de los ensayos mencionados anteriormente, se propone mejorar las condiciones de las mezclas de residuos aplicados a afirmado, subbase y terraplén, teniendo en cuenta la granulometría, índice de plasticidad, CBR y costos.

Este trabajo requiere la solución a un problema de optimización del material residual, cumpliendo tres objetivos principales. Estos objetivos están orientados a ajustar materiales residuales que cumplan con las 
condiciones para aplicarse en afirmado, subbase y terraplén, los cuales se formulan de la siguiente manera: i) encontrar la fracción adecuada de uno o más aditivos que permitan ajustar el CBR, ii) ajustar la granulometría del material y iii) mejorar la pasticidad del material, con base en los requisitos formulados por la norma INVÍAS. INVÍAS (2013)

Se propone definir un problema multiobjetivo, el cual requiere maximizar el CBR y minimizar los costos asociados al incremento del CBR y el mejoramiento de la granulometría del material; adicionalmente, hay varias restricciones tales como: i) mantener los límites granulométricos y plasticidad, ii) las fracciones granulométricas y fracciones adicionales de material complementario deben sumar la unidad y iii) el recurso disponible. El modelo matemático del problema multiobjetivo queda de la siguiente manera:

Min: $C_{T}=\sum c_{i} \cdot x_{i}$

$\operatorname{Max}: C B R_{T}=\sum C B R_{i} \cdot x_{i}$

Las restricciones para el desarrollo del algoritmo son: i) la suma de las fracciones de material que componen la muestra debe sumar la unidad, ii) el índice de plasticidad debe estar entre los rangos permitidos por la norma para los diferentes usos y iii) el costo final de la mezcla de residuos con los porcentajes de cal y cemento adicionados.

Una buena aproximación del CBR según el tipo de aditivo, se puede observar en la Tabla 2, por ejemplo para una adición de $1 \%$ de cal en el suelo, se obtiene un CBR de 14 y para una adición del $2 \%$ de cemento se obtiene un CBR entre 86 y 90.

Tabla 2: Valores de CBR para aditivos porcentuales de cal y cemento IECA (2008)

\begin{tabular}{|c|c|c|c|}
\hline$(\%)$ Cal & CBR Cal & (\%) Cemento & $\begin{array}{c}\text { CBR } \\
\text { Cemento }\end{array}$ \\
\hline 1 & 14 & 2 & $86-90$ \\
\hline 2 & 25 & 3 & 94 \\
\hline 3 & 40 & 4 & 98 \\
\hline
\end{tabular}

Hay una relación del CBR con el porcentaje de aditivo, de los que se puede obtener una función lineal independiente, así:

$$
\begin{aligned}
& C B R_{\text {cal }}=10(\% \text { cal })+5 \\
& C B R_{\text {cem }}=4(\% \text { cem })+82 \\
& C B R_{T}=\%_{\text {cal }} /\left(\%_{\text {cal }}+\%_{\text {cem }}\right) \cdot\left[10\left(\%_{\text {cal }}\right)+5\right]+\%_{\text {cem }} /\left(\%_{\text {cal }}+\%_{\text {cem }}\right) \cdot\left[4\left(\%_{\text {cem }}\right)+82\right\rceil
\end{aligned}
$$

De donde se obtiene el CBR:

$$
\begin{aligned}
& x_{1}=\%_{\text {cal }} / 100 \\
& x_{2}=\%_{\text {cem }} / 100 \\
& x_{3}=100-\%_{\text {cal }}-\%_{\text {cem }} / 100
\end{aligned}
$$

Para la cal y cemento, tambien se tiene una relación del porcentaje añadido con el límite líquido (LL), límite plástico (LP) e índice de plasticidad (IP), como se muestra en la Tabla 3, por ejemplo para una adición de $1 \%$ de cal en el suelo, se obtiene un LL de $64 \%$, LP de $40 \%$ y un IP de $24 \%$; así mismo, para una adición del $5 \%$ de cemento se obtiene un LL de $36.6 \%$, LP de 25.1 y un IP de $10.5 \%$.

Tabla 3: Valores de índice de plasticidad para aditivos porcentuales de cal (IECA, 2008) y cemento (Garzón et al., 2006)

\begin{tabular}{|l|c|c|c|c|}
\hline \multirow{3}{*}{ Material } & $(\%)$ & Límite Líquido & Límite Plástico & Índice de Plasticidad \\
\hline \multirow{3}{*}{ Cal } & 1 & 64 & 40 & 24 \\
\cline { 2 - 5 } & 2 & 62 & 47 & 15 \\
\cline { 2 - 5 } & 3 & 59 & 48 & 11 \\
\hline
\end{tabular}


Tabla 3 (continuación)

\begin{tabular}{|l|l|l|l|l|}
\hline \multirow{3}{*}{ Cemento } & 5 & 36.6 & 25.1 & 10.5 \\
\cline { 2 - 5 } & 7 & 35.9 & 24.6 & 11.3 \\
\cline { 2 - 5 } & 9 & 35.9 & 23.8 & 12.1 \\
\hline
\end{tabular}

Se puede tener una relación lineal del porcentaje de cal y cemento con respecto al índice de plasticidad, representado matemáticamente a continuación:

$I P=-6,5\left(\%_{c a l}\right)+30$

$I P=0,38(\%$ cem $)+8,62$

De donde se obtiene el IPT

$I P_{T}=\%_{c a l} /\left(\%_{c a l}+\%_{c e m}\right) *\left[-6,5\left(\%_{c a l}\right)+30\right]+\%_{c e m} /\left(\%_{c a l}+\%_{c e m}\right) *\left[0,38\left(\%_{c e m}\right)+8,62\right]$

El costo de la mezcla final también es una restricción por la disponibilidad de recursos, la cual no es ilimitada. Es decir, se cuenta con una cantidad de dinero disponible para la adquisición de los aditivos y el material para ajustar la granulometría; este dinero puede no ser suficiente para contar con las cantidades ideales de material complementario. Sin embargo, para este trabajo se calcula las cantidades óptimas sin tener en cuenta la restricción por costo, pero se incluyen los costos unitarios de los aditivos y el material complementario para mejorar la granulometría del material inicial para describir la función objetivo del costo total (Ст). A continuación se muestran en la Tabla 4 los valores del costo de cada aditivo y material seleccionado para ajustar la granulomería.

Tabla 4: Costos unitarios de los aditivos y el material seleccionado (INVÍAS, 2015)

\begin{tabular}{|c|c|c|c|}
\hline ci & Cal & Cemento & Material Seleccionado \\
\hline $\begin{array}{c}\text { Costo Unitario } \\
(\$ / \mathrm{kg})\end{array}$ & 699 & 567 & 13,29 \\
\hline
\end{tabular}

Con el análisis planteado, se puede tener el siguiente problema multiobjetivo:

|Min: $C T=\sum c_{i} * x_{i}$

$\mid \operatorname{Max}: \mathrm{CBR}_{T}=x_{1} /\left(x_{1}+x_{2}\right) *\left[1000 x_{1}+5\right]+x_{2} /\left(x_{1}+x_{2}\right) *\left[400 x_{2}+82\right]$

La selección de la población inicial fue de 10.000 individuos, a partir de la cual se generan aleatoriamente las fracciones de cal y cemento con base a las funciones objetivo y a las restricciones. Las funciones de penalización se emplean para suprimir las soluciones que violenten las restricciones, descartando los valores que estén por debajo o por encima de los rangos de índice de plasticidad para los diferentes usos establecidos en la norma (INVÍAS, 2013). La técnica utilizada es la aleatoria o ruleta, la cual corresponde a un operador genético que recoge los padres aleatoriamente para recombinarlos y generar nuevos hijos. Posteriormente, se reordenan los valores con el fin de buscar el costo mínimo con mayor valor de CBR para ello se aplicó el elitismo con un valor del 1\%, esto es, guardando el mejor individuo entre una generación y otra durante el proceso evolutivo. Finalmente, se evalúan las restricciones con base a los límites señalados y se establece un número máximo de 150 generaciones como criterio de parada.

\section{RESULTADOS}

Los resultados se van a clasificar en dos componentes: Uno que indica las características iniciales del material para establecer sus posibilidades de utilización como material en infraestructura vial y; por otro lado, en caso que las condiciones iniciales no sean las esperadas, definir las mezclas de residuos y aditivos requeridos para ajustarlo a las requisitos mínimos para ser utilizado como afirmado, subbase y terraplén.

\section{Caracterización inicial del material}

La tabla 5 revela los resultados de la caracterización inicial de las muestras de residuo tomadas de las pilas. La información de la tabla indica que el material es homogéneo, con características hidrofóbicas dada la poca área superficial y los bajos contenidos de humedad y óxido de calcio. Adicionalmente, predominan los 
óxidos de silicio, aluminio y hierro. Esto muestra que el material no cumple con los requerimientos para ser utilizado como material para afirmado, subbase y terraplén, por lo tanto, lo pasos siguientes intentan ajustar el material para que puedan ser aplicados en vías terciarias.

Tabla 5: Análisis elementales

\begin{tabular}{|c|c|c|c|c|c|c|c|c|c|}
\hline Muestras & $\begin{array}{c}\text { Densidad } \\
\text { Real } \\
\left(\mathrm{gr} / \mathrm{cm}^{3}\right)\end{array}$ & $\begin{array}{c}\text { Densidad } \\
\text { aparente } \\
\left(\mathrm{gr} / \mathrm{cm}^{3}\right)\end{array}$ & $\begin{array}{c}\text { Área } \\
\text { superficial } \\
\mathrm{BET} \\
\left(\mathrm{m}^{2} / \mathrm{g}\right)\end{array}$ & $\begin{array}{c}\text { Conductividad } \\
\text { eléctrica } \\
(\mu \mathrm{s} / \mathrm{m})\end{array}$ & $\begin{array}{c}\text { Humedad } \\
(\%)\end{array}$ & $\begin{array}{c}(\% \mathrm{P} / \mathrm{P}) \\
\mathrm{S}_{2} \mathrm{O}_{2}\end{array}$ & $\begin{array}{c}(\% \mathrm{P} / \mathrm{P}) \\
\mathrm{Al}_{2} \mathrm{O}_{3}\end{array}$ & $\begin{array}{c}(\% \mathrm{P} / \mathrm{P}) \\
\mathrm{Fe} \mathrm{O}_{3}\end{array}$ & $\begin{array}{c}(\% \mathrm{P} / \mathrm{P}) \\
\mathrm{CaO}\end{array}$ \\
\hline $\mathrm{A}$ & 2,56 & 1,6 & 6,26 & 32900 & 1,28 & 63,25 & 17,53 & 8,87 & 1,36 \\
\hline $\mathrm{B}$ & 2,64 & 1,7 & 10,17 & 41000 & 1,28 & 59,25 & 20 & 9,22 & 1,99 \\
\hline C & 2,56 & 1,49 & 10,71 & 122100 & 1,84 & 56,05 & 21,41 & 10,82 & 1,57 \\
\hline D & 2,71 & 1,74 & 5,64 & 310 & 0,5 & 62,58 & 22,82 & 5,33 & 0,08 \\
\hline E & 2,69 & 1,68 & 6,31 & 2970 & 0,9 & 62,59 & 17,23 & 7,12 & 0,61 \\
\hline
\end{tabular}

La Tabla 6 muestra la distribución granulométrica de las muestras de residuos, presentando un alto contenido de material fino, superior a $12 \%$, el cual es el límite establecido por la norma. En general los resultados obtenidos muestran que la clasificación de estos materiales como material de subrasante es regular a malo (Braja, 2015).

Tabla 6: Distribución granulométrica

\begin{tabular}{|c|c|c|c|c|}
\hline Muestras & \% Gravas & \% Arenas & \% Finos & Clasificación AASHTO \\
\hline A & 25,4 & 38,5 & 36,1 & A-4 \\
\hline B & 24,9 & 26,9 & 48,3 & A-6 \\
\hline C & 27 & 24,3 & 48,7 & A-6 \\
\hline D & 4,8 & 57,2 & 38,1 & A-4 \\
\hline E & 42,8 & 37,5 & 19,7 & A-2-6 \\
\hline
\end{tabular}

La Tabla 7 muestra los resultados de los índices de consistencia y CBR, en los cuales se observa que las muestras de residuos presentan una baja capacidad de soporte (CBR), los cuales deben ser mayores a $15 \%, 40 \%$ y $5 \%$ para afirmado, subbase y terraplén respectivamente. Por otra parte, el índice de plasticidad debe estar entre $4 \%$ y $9 \%$, mayor a $6 \%$ y mayor a $15 \%$, para afirmado, subbase y terraplén, respectivamente. En vista que el material no presenta propiedades apropiadas para ser utilizado como material de afirmado, subbase y terraplén, a continuación se plantean los resultados que ajustan estas condiciones.

Tabla 7: Análisis aproximado

\begin{tabular}{|c|c|c|c|c|}
\hline Muestras & $\begin{array}{c}\text { Límite } \\
\text { Líquido (\%) }\end{array}$ & $\begin{array}{c}\text { Límite } \\
\text { Plástico (\%) }\end{array}$ & $\begin{array}{c}\text { Índice de } \\
\text { Plasticidad (\%) }\end{array}$ & $\begin{array}{c}\text { CBR compactado } \\
\text { al 98\% }\end{array}$ \\
\hline A & 22,7 & 16,7 & 6 & 3,5 \\
\hline B & 31,8 & 18,2 & 14 & 3,4 \\
\hline C & 25 & 14,3 & 11 & 3,4 \\
\hline D & 19 & 14,5 & 5 & 5,5 \\
\hline E & 19,6 & 16,1 & 4 & 5,5 \\
\hline
\end{tabular}

Los resultados de composición mineralógica de los materiales analizados por Bastidas et al. (1993) mediante difracción de Rayos X fueron en su mayoría cuarzo, caolinita, ilita y feldespatos; respecto al índice de plasticidad se obtuvieron resultados entre 10 y 30, y LL entre 40 y 60 . De acuerdo a los resultados; las arcillas corresponden a un tipo de arcillas caolíniticas de alta plasticidad, arcillas mixtas caolino-ilíticas de alto contenido en fracción de arcilla y bajo contenido de carbonatos y $\mathrm{Fe}_{2} \mathrm{O}_{3}$ y arcillas mixtas iliticocaoliniticas ferruginosas presentando contenidos variables de cuarzo y de compactación baja o media. Considerando el índice de plasticidad presentado anteriormente, está muy relacionado con el estéril de la 
mina Cerrolargo, presentado en este trabajo; por lo tanto, se aprecia que es necesario un proceso de estabilización con cal o cemento para ser utilizado como material de afirmado

\section{Optimización del material para ser aplicado como afirmado, subbase y terraplén}

La Figura 2 muestra el ajuste granulométrico de las mezclas de residuos con material seleccionado procedente de la misma mina. En dicho ajuste se observa que para que la mezcla cumpla como material de afirmado, se requiere una relación $80 / 20$ y para material de subbase una relación $80 / 15$, es decir; $80 \%$ de material seleccionado y 20 y $15 \%$ de residuos mineros respectivamente. En lo que respecta a los requisitos mínimos de los agregados para ser utilizados como material de terraplén, la norma establece un tamaño máximo de $100 \mathrm{~mm}$, mientras que los por porcentajes que pasa el tamiz No.10 y el tamiz No. 200 debe ser de $80 \%$ y $35 \%$ máximo para cada uno de los tamices. Según resultados obtenidos en ensayos de laboratorio las muestras de residuos mineros cumplen con estos requisitos.

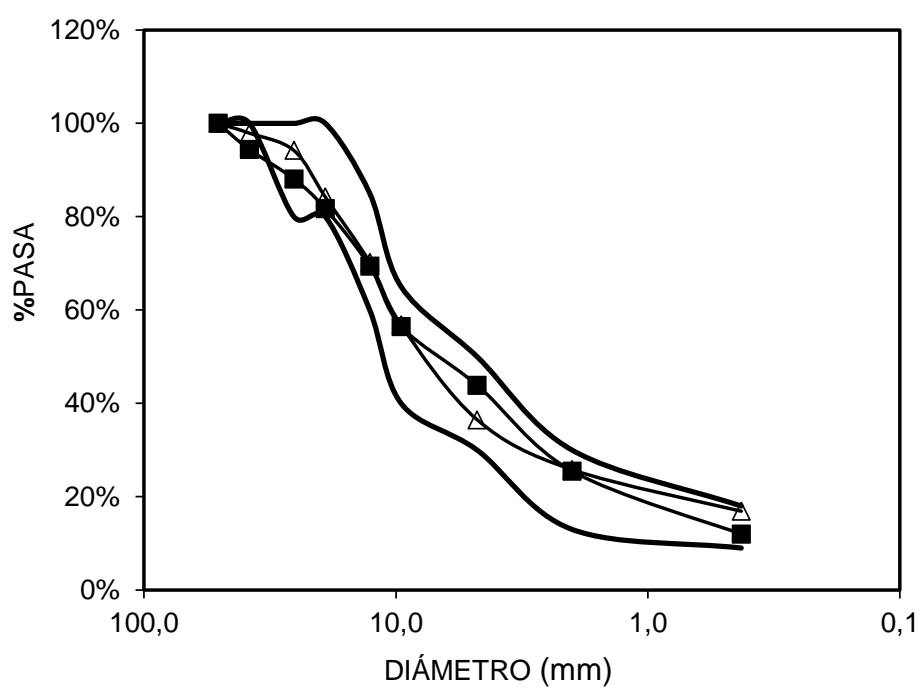

a.

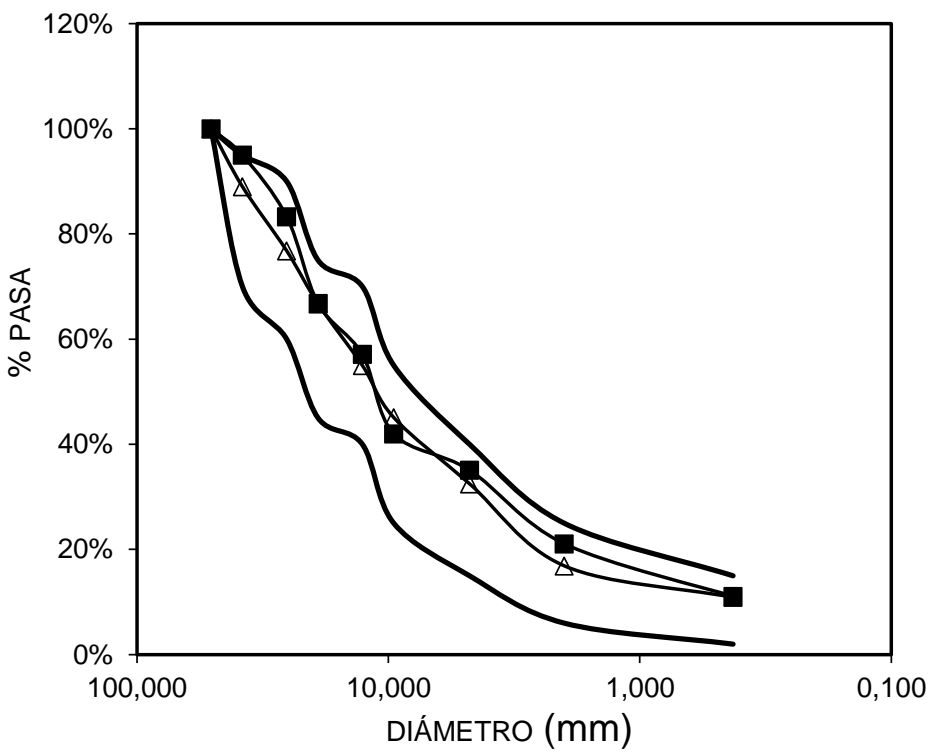

b.

Fig. 1: Ajuste granulométrico para material de afirmado (a) y subbase (b). Los símbolos sin relleno son valores experimentales $(\Delta)$ y los con relleno son calculados

La Figura 3, se aprecia la curva de Pareto para material de afirmado, donde los puntos oscuros hacen parte de la frontera de Pareto o puntos óptimos obtenidos por el algoritmo y los puntos verdes corresponden a todos los puntos alcanzados durante las generaciones evaluadas. La curva muestra muchos valores óptimos, entre los que puede ocurrir dos escenarios a saber: i) que el evaluador requiera minimizar los costos, lo que acarrea que el CBR sea mínimo o ii) que busque un punto medio entre el CBR y los costos del material. Estas mismas condiciones se aplicaron para ajustar las mezclas de residuos para ser utilizados como material de subbase y terraplén como se muestra en la Tabla 8. 


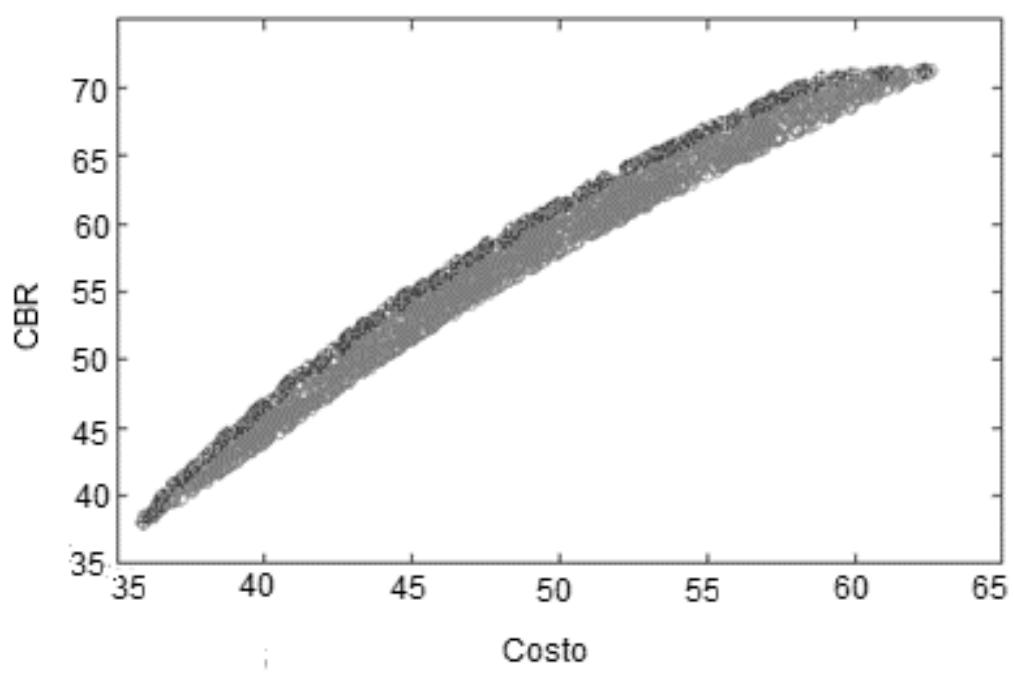

Fig. 2: Curva Pareto óptima de las F1 (CBR) y F2 (Costos) para Afirmado

Tabla 8: Resultados de la evaluación multiobjetivo para material de afirmado y subbase

\begin{tabular}{|c|c|c|c|c|c|c|}
\hline Uso & \multicolumn{2}{|c|}{ Afirmado } & \multicolumn{2}{c|}{ Subbase } & \multicolumn{3}{c|}{ Terraplén } \\
\hline Ítem & Escenario i & Escenario ii & Escenario i & Escenario ii & Escenario i & Escenario ii \\
\hline Fracción Cal & 0,03 & 0,03 & 0,04 & 0,04 & 0,0282 & 0,0398 \\
\hline Fracción Cemento & 0,00 & 0,02 & 0,00 & 0,01 & 0,0003 & 0,0244 \\
\hline Mezcla 80/20 & 0,97 & 0,94 & 0,96 & 0,94 & 0,9997 & 0,9994 \\
\hline CBR (\%) & 38,96 & 61,24 & 42,26 & 54,64 & 33,59 & 62,65 \\
\hline Costo $(\$ / \mathrm{kg})$ & 36,20 & 49,93 & 38,80 & 47,03 & 32,74 & 54,10 \\
\hline IP $(\%)$ & 8,83 & 8,85 & 5,96 & 5,92 & 11,67 & 6,18 \\
\hline
\end{tabular}

\section{Validación del problema de optimización}

Para el proceso de validación se realiza una mezcla de materiales con el fin de ajustar la granulometría, pues este tipo de material por sí solo no cumple con los requisitos establecidos en norma INVÍAS para afirmado, subbase y terraplén. Para ello se consideró inicialmente una mezcla $50 / 50$, es decir, el $50 \%$ de los residuos mineros proveniente de la extracción de carbón y el 50\% restante material de la misma mina pero que ha sido sometido a un proceso de zarandeo y tamizado que permita eliminar los sobre tamaños, es decir, partículas mayores a 4". Seguidamente se adicionan los porcentajes de cal y cemento resultantes del desarrollo del AEMO para los diferentes usos. Con la mezcla mencionada anteriormente se realizaron los ensayos de granulometría, límites de Atterberg, Proctor modificado y CBR, Tabla 9.

Tabla 9: Comparativo entre los resultados de la evaluación multiobjetivo y la validación.

\begin{tabular}{|c|c|c|c|c|c|c|}
\hline Uso & \multicolumn{2}{|c|}{ Afirmado } & \multicolumn{2}{c|}{ Subbase } & \multicolumn{2}{c|}{ Terraplén } \\
\hline Resultado & AEMO & Validación & AEMO & Validación & AEMO & Validación \\
\hline Índice de plasticidad & 8,83 & 8,23 & 5,96 & 5,65 & 11,67 & 11,10 \\
\hline CBR (\%) & 38,96 & 31,93 & 42,26 & 56,29 & 33,59 & 14,27 \\
\hline
\end{tabular}

De la Tabla 9, se calcularon los porcentajes de error, arrojando valores de $7 \%, 5 \%$ y $5 \%$; para el índice de plasticidad, mientras que para el CBR se obtuvieron porcentajes elevados, esto es $18 \%, 25 \%$ y $58 \%$ para los diferentes usos, es decir afirmado, subbase y terraplén respectivamente. Sin embargo, este parámetro en la validación, para todos los casos, se encuentra por encima de los requisitos mínimos establecidos por la norma. Bravo (2012) analiza la viabilidad de la adición de dos tipos de Materiales de Excavación de Reutilización Industrial (MERI) de minas, este análisis experimental expone las técnicas de recolección y caracterización de las muestras de MERI, las cuales también fueron tomadas de las capas superficiales y se aplicaron las mismas técnicas de difracción de Rayos $\mathrm{X}$ y granulometría para caracterización física y química de estos materiales. Esta investigación fue realizada con MERI procedentes de dos minas de España, sin embargo, al comparar los resultados obtenidos mediante difracción de rayos $\mathrm{X}$ se tiene que las 
principales fases cristalinas encontradas también corresponden a cuarzo y caolinita. Es importante destacar que los resultados presentados por Bravo, muestran la posibilidad de dosificar las muestras de material para darle características de revestimiento y mejorar la capacidad de afirmado. El modelo propuesto por Trivedi et al (2013), solo se limita a predecir los valores de CBR del suelo con base a los datos experimentales, por tanto, la tabla 10 muestra los datos comparativos para esta variable, con los resultados obtenidos de las mezclas con cal y cemento para ser utilizados como material de subbase y los obtenidos por Trivedi et al (2013), quienes analizan un tipo de suelo para el mismo uso mediante la adición de un $20 \%$ de cenizas volantes.

Tabla 10: Comparativo entre los resultados de CBR obtenidos en la validación y Trivedi et al (2013)

\begin{tabular}{|c|c|c|}
\hline Referentes & Método de Cálculo & CBR (\%) \\
\hline Autores & AEMO & 42,26 \\
\cline { 2 - 3 } (Subbase) & Experimental & 56,29 \\
\hline Trivedi et al (2013) & Predicho & 20,42 \\
\cline { 2 - 3 }$(20 \%$ de cenizas) & Experimental & 20,53 \\
\hline
\end{tabular}

Se observa, que aplicando directamente $4 \%$ de cal y $0,02 \%$ de cemento los porcentajes de CBR se incrementa en un $56,29 \%$ con respecto al uso directo de cenizas volantes que solamente alcanza un $20,53 \%$ posiblemente debido a que este material contiene componentes adicionales al calcio como el dióxido de silicio $\left(\mathrm{SiO}_{2}\right)$, óxido de aluminio $\left(\mathrm{Al}_{2} \mathrm{O}_{3}\right)$ y óxido de hierro $\left(\mathrm{Fe}_{2} \mathrm{O}_{3}\right)$.

\section{CONCLUSIONES}

De los resultados mostrados, de su análisis y de su discusión, se pueden obtener las siguientes conclusiones: 1) la herramienta desarrollada mediante el uso de Algoritmos Evolutivos (AEMO) permite obtener los porcentajes óptimos de cal y cemento que se deben adicionar a mezclas de residuos para ser utilizados como material de afirmado, subbase y terraplén; 2) la formulación del Algoritmo Evolutivo (AEMO) permitió mediante la definición de las funciones objetivo, restricciones y variables de optimización, obtener las mezclas óptimas de mezclas residuos y los porcentajes mínimos de cal y cemento que se deben adicionar a este tipo de mezclas para ser utilizados como material de afirmado, subbase y terraplén; y 3 ) esta herramienta puede ser utilizada en obras civiles de gran envergadura con el fin de evitar posibles errores al momento de realizar la mezcla de residuos junto con las proporciones mínimas de cal y cemento necesarias para mejorar las propiedades de los mismos y cumplir con las exigencias de la norma.

\section{NOTACIONES}

$\begin{array}{ll}C_{T} & =\text { Costos totales } \\ C B R & =\text { Capacidad de soporte del suelo } \\ c i & =\text { Costo de cada uno de los materiales } \\ I P & =\text { Índice de plasticidad } \\ C B R_{T} & =\text { CBR totales } \\ C B R i & =\text { CBR de cada uno de los materiales } \\ i & =\text { Fracciones de cal y cemento } \\ x_{i} & =\text { Fracciones de mezcla final } \\ x_{1} & =\text { Fracción de cal } \\ X_{2} & =\text { Fracción de cemento } \\ X_{3} & =\text { Fracción de material seleccionado } \\ I P_{T} & =\text { Índice de plasticidad total }\end{array}$

\section{REFERENCIAS}

Asociación Nacional de Fabricantes de Cales y Derivados de España (ANCADE), Asociación Nacional Técnica de Estabilizados de Suelos y Reciclado de Firmes (ANTER) e Instituto del Cemento y sus Aplicaciones (IECA). Manual de estabilización de suelos con cemento o cal, Madrid (2008)

Bastidas, J., A. López, J. Serrano, J. Torre y M. Signes, Mineralogía aplicada de arcillas constitutivas de estériles en minas de carbón de la zona minera de Teruel, Cuadernos de Geología Ibérica, (17), 153-169 (1993)

Bastidas, M., R. Bermúdez, G. Jaramillo y F. Chejne, Optimización termoeconómica y ambiental usando algoritmos genéticos multiobjetivo, doi: 10.4067/S0718-07642010000400006, Información Tecnológica, 21(4), 35-44 (2010)

Bravo R.J., Incorporación de residuos mineros a materiales de construcción (Doctoral dissertation, Arquitectura Técnica) (2012) 
Chau, K.W. y F. Albermani, Knowledge-based system on optimum design of liquid retaining structures with genetic algorithms, doi 10.1061/(ASCE)0733-9445(2003)129:10(1312), J. of structural engineering, 129(10), 1312-1321 (2003)

Chisari, C., C. Bedon y C. Amadio, Dynamic and static identification of base-isolated bridges using Genetic Algorithms, doi 10.1016/j.engstruct.2015.07.043, Engineering Structures, 102, 80-92 (2015)

Coello, C. C., A. Christiansen y F. Hernández, A simple genetic algorithm for the design of reinforced concrete beams, doi: 10.1007/BF01200046, Engineering with Computers, 13(4), 185-196 (1997)

Das, B.M., Principios de ingeniería de Cimentaciones, 4를. Thomson SA, México (2001)

Đumić, M., D. Šišejković, R. Čorić y D. Jakobovi, Evolving priority rules for resource constrained project scheduling problem with genetic programming, doi: 10.1016/j.future.2018.04.029, Future Generation Computer Systems, 86, 211$221(2018)$

Fairbairn, E.M., M. Silvoso, R. Toledo, J. Alves y N. Ebecken, Optimization of mass concrete construction using genetic algorithms, doi: 10.1016/j.compstruc.2003.08.008, Computers \& structures, 82(2), 281-299 (2004)

Fernández, L. V., Manual de reutilización de residuos de la industria minera, siderometalúrgica y termoeléctrica, Instituto Tecnológico Geominero de España, Madrid (1995)

Gandomi, A. y A. Kashani, Automating pseudo-static analysis of concrete cantilever retaining wall using evolutionary algorithms, doi: 10.1016/j.measurement.2017.10.032, Measurement, 115, 104-124 (2008)

Garzón, E., P. Sánchez y A. Ruiz, Efectos de la compactación y estabilización con cemento y cal en las propiedades mecánicas de filitas, XXVI REUNIÓN (SEM)/XX REUNIÓN (SEA), 217-220 (2006)

INVÍAS Manual de Diseño Geométrico de Carreteras, Ministerio de Transporte, Instituto Nacional de Vías, Bogotá (2008) INVÍAS, Análisis de precios unitarios, Territorial Cundinamarca, Bogotá (2015)

Kanumuri, L., D. Pushpalatha, A. Naidu y S. Singh, A Hybrid Neural Network-Genetic Algorithm for Prediction of Mechanical Properties of ASS-304 at Elevated Temperatures, doi: 10.1016/j.matpr.2017.01.081, Materials Today: Proceedings, 4(2), 746-751 (2017)

Korkana, P., V. Kanakoudis, M. Patelis y K. Gonelas, Forming District Metered Areas in a Water Distribution Network Using Genetic Algorithms, doi: 10.1016/j.proeng.2016.11.095, Procedia Engineering, 162, 511-520 (2016)

Lee, C., y J. Ahn, Flexural design of reinforced concrete frames by genetic algorithm, doi: 10.1061/(ASCE)07339445(2003)129:6(762) Journal of structural engineering, 129(6), 762-774 (2003)

Lepš, M., y M. Šejnoha, New approach to optimization of reinforced concrete beams, doi: 10.1016/S00457949(03)00215-3, Computers \& structures, 81(18), 1957-1966 (2003)

Lim, C.H., Y.S. Yoon y J.H. Kim, Genetic algorithm in mix proportioning of high-performance concrete, doi: 10.1016/j.cemconres.2003.08.018, Cement and Concrete Research, 34(3), 409-420 (2004)

Mas, M., E. García, L. Marco y J. Marco, Análisis de la Viabilidad Ambiental de la Utilización de Morteros Fabricados con Polvo de Vidrio en la Estabilización de Suelos, doi 10.4067/S0718-07642016000500010, Información Tecnológica, 27(5), 77-86 (2016)

Morales, D., Valoración de las cenizas de carbón para la estabilización de suelos mediante activación alcalina y su uso en vías no pavimentadas, BS Tesis, Facultad de Ingeniería, Universidad de Medellín, Medellín (2015)

Ramírez, S., Metodología basada en Algoritmos Genéticos y Programación en Paralelo para el Diseño Óptimo de Armaduras de Acero, Tesis de maestría, Facultad de Ingeniería, Universidad Javeriana, Bogotá (2014)

Rojas L., J. López y N. Muñoz, Asignación Óptima de Presupuesto para Mejoramiento de la Calidad del Servicio en Sistemas de Distribución usando Algoritmo Genético No-Dominado II (NSGA-II) y un Algoritmo Memético, doi 10.4067/S0718-07642016000100013, Información Tecnológica, 27(1), 115-126 (2016)

Saffon, C., Programación de proyectos de construcción de edificaciones mediante un algoritmo genético, Tesis de Licenciatura, Facultad de Ingeniería, Pontificia Universidad Javeriana, Bogotá (2005)

Taskiran, T., Prediction of California bearing ratio (CBR) of fine grained soils by Al methods, doi: 10.1016/j.advengsoft.2010.01.003, Advances in Engineering Software, 41(6), 886-892 (2010)

Tiachacht, S., A. Bouazzouni y otros cuatro autores, Damage assessment in structures using combination of a modified Cornwell indicator and genetic algorithm, doi: 10.1016/j.engstruct.2018.09.070, Engineering Structures 177 (2018): 421 $430(2018)$

Torres, C., Optimización heurística multiobjetivo para la gestión de activos de infraestructuras de transporte terrestre, Tesis Doctoral, Facultad de Ingeniería, Pontificia Universidad Católica de Chile, Santiago de Chile (2015)

Trivedi, J., S. Nair y C. Iyyunni. Optimum utilization of fly ash for stabilization of sub-grade soil using genetic algorithm, doi: 10.1016/j.proeng.2013.01.034, Procedia Engineering, 51, 250-258 (2013) 